Similarly for species $B$. If the surface is saturated, the time during which a centre is left vacant is negligible compared with the time it is covered, and

$$
t=\frac{\mu_{\mathbf{A}} \tau_{\mathbf{A}} p n+\mu_{\mathrm{B}} \tau_{\mathbf{B}}(\mathrm{I}-p)}{\mu_{\mathbf{A}} p+\mu_{\mathbf{B}}(\mathrm{I}-p)} .
$$

Thus the fraction of the total time the centre of activity is occupied by the reactant is

$$
\frac{\mu_{\mathbf{A}} \tau_{\mathbf{A}} p}{\mu_{\mathbf{B}} \tau_{\mathbf{B}}(\mathrm{I}-p)+\mu_{\mathbf{A}} \tau_{\mathbf{A}} p}=v
$$

and is the fractional reduction in the reaction velocity. Thus

$$
\mathbf{I} / v=(\mathbf{I}-\lambda)+\lambda / p,
$$

where $\lambda=\sqrt{\frac{M_{\mathrm{A}}}{\bar{M}_{\mathrm{B}}} \cdot \frac{\tau_{\mathrm{B}}}{\tau_{\mathbf{A}}} \text { and } M_{\mathbf{A}} \text { represents the molecular }}$ weight, and $\tau_{\mathbf{A}}$ the mean life of the molecule $A$ on the surface. This formula may be written

$$
(\mathrm{I}-v) / v=\frac{\tau_{B}}{\tau_{\mathbf{A}}} \cdot \frac{\mu_{B}(\mathrm{I}-p)}{\mu_{\mathrm{A}} p},
$$

and the fraction $(I-v) / v$ is the ratio of the number of molecules of $B$ to the number of molecules of $A$ present on the surface. $\mu_{B}(x-p) / \mu_{A} . p$ is the ratio of the number of molecules of $B$ to $A$ bombarding the surface $=n_{B} / n_{A}$. Thus if $n_{B} / n_{A}$ be multiplied by the ratio of the mean lives of molecules $B$ to $A$ on the surface, we obtain the composition of the adsorbed gas film for saturated surfaces. The composition of the adsorbed gas film in equilibrium with a mixture of gases $A$ and $B$ is given by

$$
\frac{x_{\mathrm{A}}}{x_{\mathrm{A}}+x_{\mathrm{B}}}=\frac{\mathrm{I}}{\mathrm{I}+\sqrt{\frac{\bar{M}_{\mathrm{A}}}{M_{\mathrm{B}}} \frac{\tau_{\mathrm{B}}}{\tau_{\mathrm{A}}} \frac{p_{\mathrm{B}}}{p_{\mathrm{A}}}}} .
$$

These formulæ have been verified for partial pressures of alcohol in water varying from 0.02 to $\mathrm{I} \cdot \mathrm{OO}$ times the total pressure.

\begin{tabular}{|c|c|c|}
\hline $\begin{array}{c}\text { Fractional Reaction } \\
\text { Velocity observed. } \\
v .\end{array}$ & $\begin{array}{c}\text { Calculated from } \\
\text { [I } / v=0.64+0.36 / p .\end{array}$ & $\begin{array}{c}\text { Fractional partial Pressure } \\
\text { of Alcohol in Alcohol Water } \\
\text { Mixtures }=p \text {. }\end{array}$ \\
\hline & 1.00 & $\mathbf{I} \cdot 00$ \\
0.94 & 0.92 & 0.80 \\
0.83 & 0.81 & 0.60 \\
0.60 & 0.65 & 040 \\
0.40 & $0.4 \mathrm{I}$ & 0.20 \\
0.22 & 0.24 & 0.10 \\
0.10 & 0.13 & 0.05 \\
0.05 & 0.05 & 0.02 \\
\hline
\end{tabular}

The agreement between the observed and calculated values is within the limits of experimental error.

The apparatus with which these measurements were made has already been described (Proc. Camb. Phil. Soc., vol. 22, pp. $73^{8-50}$ ) and is very convenient for obtaining the wide variations in partial pressure necessary.

St. John's College,

F. H. Constable.

Cambridge, July 30.

\section{On the Origin of Species in Flowering Plants:}

THE origin of species is the origin of the difference between two species. The existence of a peculiar difference between good species, which does not exist between varieties, has long been realised by systematists and others. This essential difference expresses itself, in part, in flowering plants, in the abortion of pollen and ovules in the hybrids, which springs, in typical cases: (a) from a different distribution of genes between two or more chromosome pairs; so that vital genes are omitted after the reduction division in some or all of the groups of chromosomes of the $F_{1}$ of the cross. An example of this is probably afforded by the crosses of certain species of Stizolobium (Mucuna) (Zeitsch. f. ind. Abst. u. Vererb., pp. 303-342, I9 4 ), two non-homologous chromosomes appearing to have interchanged genes in one of the species. In the second case $(b)$ the attraction between chromosomes which should be homologous fails in the reduction divisions of the $F_{1}$ plant, so that non-conjunction (Journal of Genetics, I925) takes place with regard to one or more pairs. Thus there is an absence of vital chromosomes or the presence of extra chromosomes in some of the pollen and ovules. This is illustrated by the two cannas "Austria" and "Italia," which belong no doubt, as their history states, to the $F_{1}$ of species crosses. In "Austria," for example, the I8 single chromosomes show only I-3 pairs at the reduction divisions. All the other chromosomes segregate at random, so that no more than one pollen-grain or embryo-sac out of thousands contains only the 9 chromosomes from one parent, and survives. In the cross of radish and cabbage, as Karpechenko (Journal of Genetics, vol. I4, pp. 375-396, r924) has lately shown, chromosome conjugation is absent. This has also been shown in certain Digitalis crosses.

Hence the origin of species is effected, inter alia, by $(a)$ segmental interchange between non-homologous chromosomes, of which some evidence has lately been gathered in Datura, and $(b)$ by the loss of attraction between chromosomes which were homologous, producing non-conjunction (Journal of Genetics, 1925). The hypothesis of the origin of species from crosses between species in a genus posits the existence of allied species, and can thus only be applicable in more or less exceptional cases.

The species listed in any genus may consist of forms each showing one or more of the following differences from the nearest allied species :

(I) Mendelian differences in one or more pairs of genes ;

(2) Mendelian or other differences permanently sustained by propagation by apogamy;

(3) Shifting of some genes in one species between two or more non-homologous chromosomes;

(4) Loss of mutual attraction between two or more corresponding chromosomes in the two species;

(5) Different chromosome numbers or sizes.

Of course the mere possibility or impossibility of securing an $F_{1}$ between two species may often be due to other more superficial causes than these fundamental differences.

Carnegie Institution of Washington.

John Belling.

\section{The Structure of Benzene and Gyclohexane and their Optical Anisotropy.}

IN a recent paper (Roy. Soc.-Proc., A, vol. Io7, p. 684), I have shown that the optical anisotropy of the gaseous molecules of a number of di-atomic and tri-atomic compounds which is evidenced by the polarisation of the light scattered from them, can be explained by the mutual action of the electrical doublets induced in the different atoms of the molecule by the electric field of the incident radiation. Each atom was assumed to behave isotropically. Satisfactory agreement was obtained between the observed and calculated values of the depolarisation of the scattered light in the case of the molecules $\mathrm{CO}_{2}, \mathrm{~N}_{2} \mathrm{O}$ and $\mathrm{CS}_{2}$. 